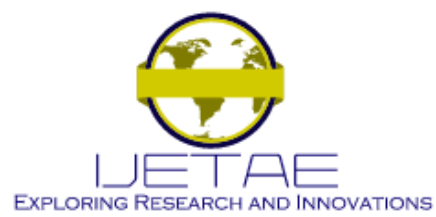

International Journal of Emerging Technology and Advanced Engineering

Website: www.ijetae.com (ISSN 2250-2459, ISO 9001:2008 Certified Journal, Volume 10, Issue 08, August 2020)

\title{
An ANN Ensemble Based ECG Signal Classification Approach for Accurate Arrhythmia Detection
}

\author{
Gaddam Chandra Mohan ${ }^{1}$, Shyam Sundar Pattnaik ${ }^{2}$ \\ ${ }^{I}$ Research Scholar, ECE Deptt, NITTTR, Chandigarh, India \\ ${ }^{2}$ Director, NITTTR, Chandigarh, India
}

\begin{abstract}
Artificial Neutral Networks (ANN) based classification of the electrocardiogram signal to identify various diseases of the heart is a popular method. However, as the classification error can lead to fatal consequences, so the new approaches to improve the accuracy of ANN based methods has been an active area of research during the past decade. An ANN ensemble based approach to detect the arrhythmia from ECG signal by classifying into normal and abnormal classes has been proposed in this presented work. The high accuracy of classification has been observed from the results which makes the proposed approach very effective. Another advantage of the proposed approach is its straightforward design which makes it very easy for implementation.
\end{abstract}

Keywords-- ANN, ANN Ensemble, ECG Classification, Arrhythmia Detection

\section{INTRODUCTION}

The working of the human body system involves synchronized working of many sub systems like nervous system, cardio vascular system, musculo-skeleton system etc. All sub-systems work continuously and produce electrical signals known as Bio-Medical Signals. These signals are measured by doctor with the help of various instruments and then analyzed to diagnose any disease. The electrocardiogram (ECG) is a signal which is acquired from the heart to check its functioning and a typical ECG signal is shown in Fig. 1. ECG signal is a composite signal consisting of sub-signals named as $\mathrm{P}, \mathrm{Q}, \mathrm{R}, \mathrm{S}, \mathrm{T}$ and $\mathrm{U}$ waves having frequencies lying between 0.05 and $150 \mathrm{~Hz}$ [1]. The heart has four chambers/parts [2] and the ECG signal consists of $\mathrm{P}, \mathrm{Q}, \mathrm{R}, \mathrm{S}, \mathrm{T}$ and $\mathrm{U}$ waves represents functions of various parts of the heart. The electrical functioning of atria, the upper heart chamber, is characterized by $\mathrm{P}$ wave. The electrical functioning of ventricles, the lower heart chamber, is characterized by QRS complex. The contraction of the ventricles is signified by ST segment and similarly, the $\mathrm{T}$ wave represents the electricity flow during relaxation of the lower heart and also the information regarding the readiness for the next contraction cycle.
The ECG test is a very popular and economic test used by the doctors to diagnose various heart diseases by analyzing various parameters of this signal [3]. The common problems are that a wave can be missing or an extra pulse may exist. Also the blockage in the heart reduces the conduction and the ECG wave's amplitude gets reduced. The shape of the ECG signal and the timing relationship between various parts are the basis of different interpretation regarding the functioning of heart. Commonly, the ECG signal has been used to detect the Rhythm defects (Arrhythmia), Enlargement of the heart, Injury to the heart, Conduction defects (Blockage), etc.

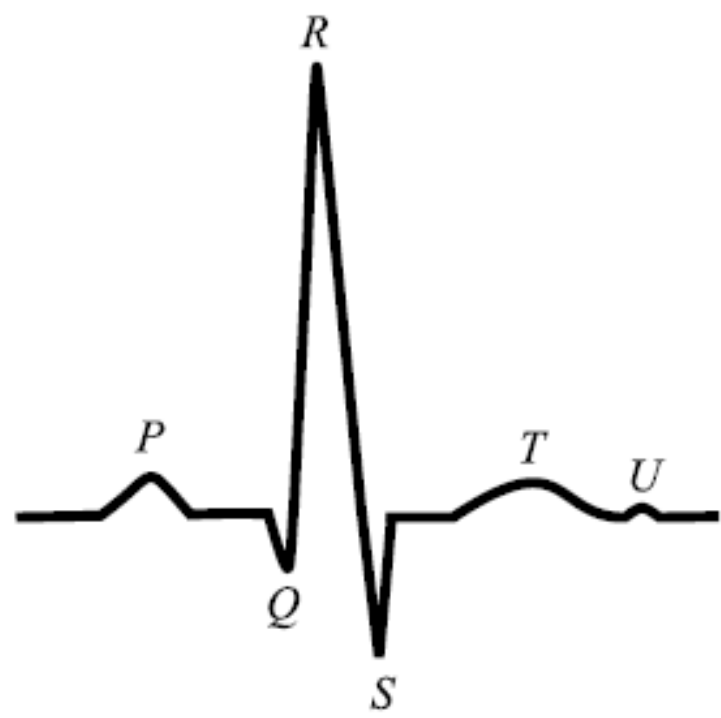

Fig. 1 A typical normal ECG signal [1]

Various scientists and researchers have employed the classification techniques to analyze the ECG signals to identify the various diseases of the heart. Artificial Neutral Networks (ANN) based approaches have also been used by various researchers for the diagnostic classification of ECG signals. The investigation of ANN performance for ECG classification by [4] is a part of early studies related to ANN application. 


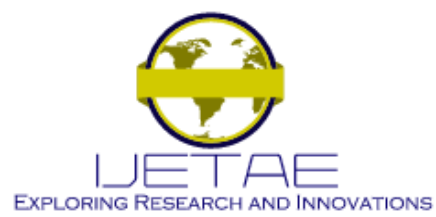

International Journal of Emerging Technology and Advanced Engineering

Website: www.ijetae.com (ISSN 2250-2459, ISO 9001:2008 Certified Journal, Volume 10, Issue 08, August 2020)

Oien et al. [5] presented the application of ANN for the 3-class problem of ECG rhythm classification by distinguishing the normal sinus rhythm and two different abnormal rhythms. Many ANN applications have also been proposed in recent years related to ECG classification. Arrhythmia, a common abnormality, has been analyzed by [6] using ANN. The heart abnormalities have been classified using the back propagation trained feed-forward ANN by [7]. In [8], different kinds of arrhythmia have been classified by employing an ANN based technique which exploits morphological and dynamic features. The classification of different categories of cardiac arrhythmias has also attracted the use of advanced ANN based algorithms such as Deep Neural Networks [9 -10], Deep Convolutional Neural Networks (CNN) [11], hybridization of CNN and Recurrence Complex Networks [12], etc. The use of a hybrid approach combining learning approaches like the deep/ensemble learning, and evolutionary optimization techniques to enhance the accuracy of arrhythmia detection has been recently reported by [13]. In this presented work, the ANN ensemble based approach has been employed to accurately detect the arrhythmia from ECG signal.
The main benefit of the presented approach is its straightforward design and uncomplicated realization. The section-2 of the paper describes the design of proposed ANN ensemble based approach. The results have been discussed in section-3 and the section- 4 presents the conclusion of the proposed work.

\section{Ann ENSEMble DeVelopment}

ANN models have been employed by various researchers for ECG classification as discussed above. However, the use of ANN ensemble approach is preferred over individual ANN models due to their several limitations which include nonspecific generalization, unsatisfactory performance while dealing with complex/ insufficient data sets [14-16]. The ANN ensemble approach makes use of multiple ANN simultaneously by combining their outputs to generate the final single output [17]. In this paper also, an ANN ensemble has been designed for the diagnostic classification of ECG signal for detection of Arrhythmia disease by classifying the ECG signal to normal and abnormal class. The proposed ensemble makes use of 10 Multilayer Perceptron (MLP) ANNs in parallel as depicted in block diagram of the model presented in Fig. 2.

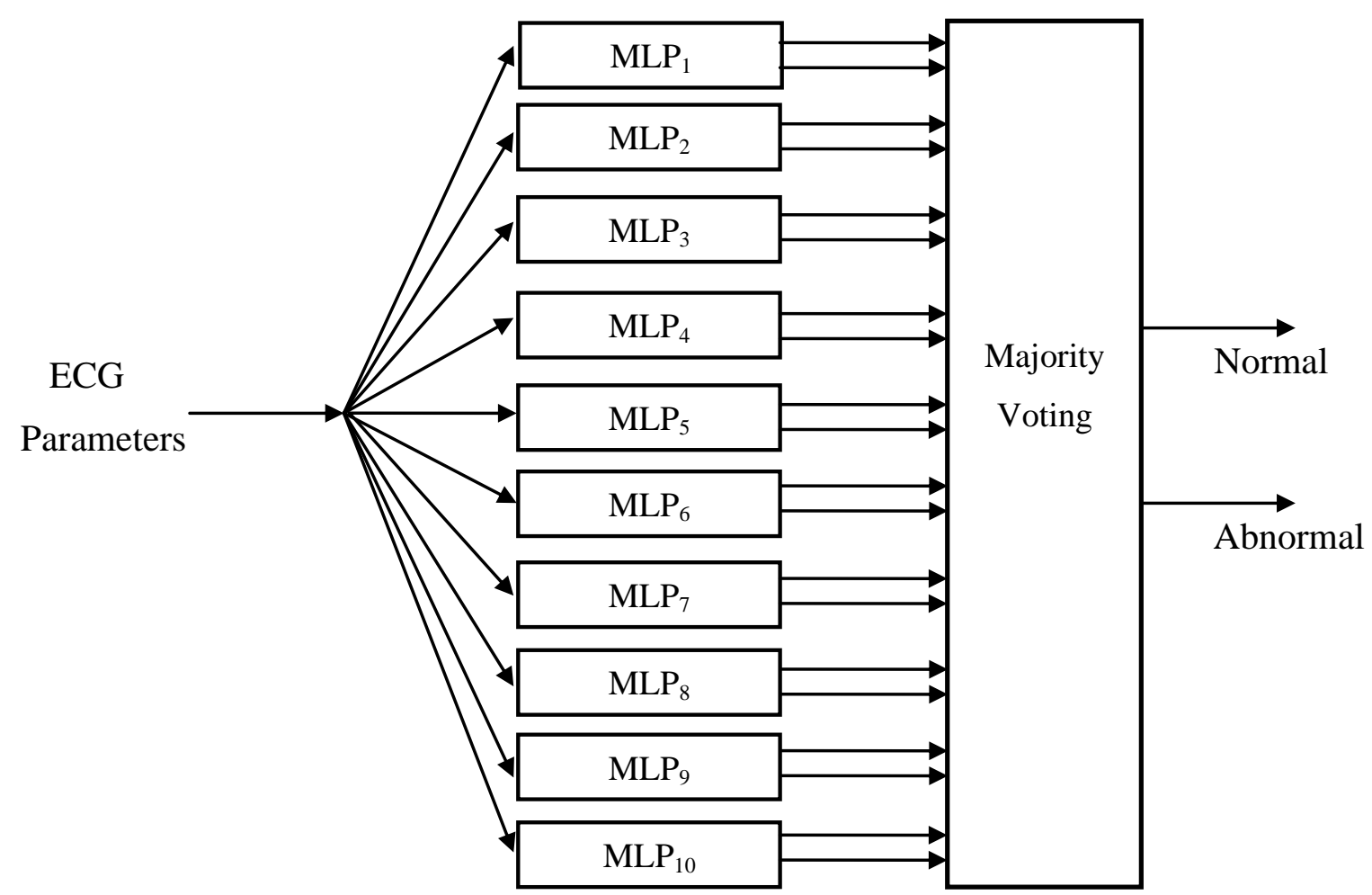

Fig. 2 Block diagram of the MLP based ANN Ensemble 


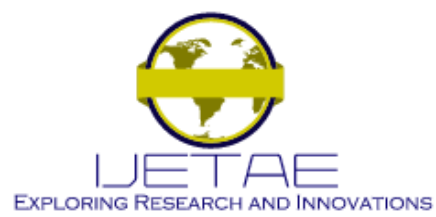

International Journal of Emerging Technology and Advanced Engineering

Website: www.ijetae.com (ISSN 2250-2459, ISO 9001:2008 Certified Journal, Volume 10, Issue 08, August 2020)

The ANN ensemble development involves three steps [18]: (i) Selection of different data sub-sets from the source data-set, (ii) Design of ANN ensemble constituents and (iii) merging of outputs of constituents to generate final ensemble output. The 60 values of source data set containing normal and abnormal traces have been extracted from MIT-BIH Arrhythmia database out of which 20 values have been used for test data set and 40 data values have been selected for training of ANN ensemble constituents. The Bagging (abbreviation for bootstrap aggregating) technique [14], one of the popular techniques, has been employed to create 10 diverse training data subsets of size in between 25-30 from the whole training data set.
The second step involves the development of 10 accurate and diverse ANN constituents. The diversity has been introduced among ensemble constituents by employing different approaches viz. training on different data subsets, different initials weights for different ensemble constituents, and dissimilar architecture i.e. having varied number of hidden layers and neurons in these hidden layers. Table 1 depicts the details of the structures of various ANN ensemble constituent members. All constituents have been trained to meet the performance goal of $10^{-6}$ to achieve the accuracy which is the second desired characteristic of this ensemble design step. The 10 different outputs generated by the constituents have been combined to produce the overall output of ensemble by using the ensembling strategy of majority voting.

Table 1

Details of the structures of ANN ensemble constituent members

\begin{tabular}{|c|c|c|c|c|}
\hline $\begin{array}{c}\text { Sl. } \\
\text { No. }\end{array}$ & $\begin{array}{c}\text { Name of } \\
\text { Constituent } \\
\text { Model }\end{array}$ & $\begin{array}{c}\text { Hidden } \\
\text { layers } \\
\text { Count }\end{array}$ & $\begin{array}{c}\text { Hidden } \\
\text { neurons } \\
\text { Count }\end{array}$ & Miscellaneous parameters \\
\hline 1 & $M L P_{1}$ & 2 & 15,20 & $\begin{array}{c}\text { Training_function }=\text { Trainlm } \\
\text { Learning_Rate }=0.15\end{array}$ \\
\hline 2 & $\mathrm{MLP}_{2}$ & 1 & 25 & $\begin{array}{c}\text { Training_function }=\text { Trainlm } \\
\text { Learning_Rate }=0.2\end{array}$ \\
\hline 3 & $\mathrm{MLP}_{3}$ & 3 & $15,10,15$ & $\begin{array}{c}\text { Training_function }=\text { Trainbfg } \\
\text { Learning_Rate }=0.15\end{array}$ \\
\hline 4 & $\mathrm{MLP}_{4}$ & 2 & 20,20 & $\begin{array}{c}\text { Training_function }=\text { Traincgf } \\
\text { Learning_Rate }=0.2\end{array}$ \\
\hline 5 & $\mathrm{MLP}_{5}$ & 3 & $13,15,12$ & $\begin{array}{c}\text { Training_function }=\text { Trainlm } \\
\text { Learning_Rate }=0.2\end{array}$ \\
\hline 6 & $\mathrm{MLP}_{6}$ & 2 & 20,15 & $\begin{array}{c}\text { Training_function }=\text { Trainlm } \\
\text { Learning_Rate }=0.15\end{array}$ \\
\hline 7 & $\mathrm{MLP}_{7}$ & 3 & $15,15,15$ & $\begin{array}{c}\text { Training_function }=\text { Trainbfg } \\
\text { Learning_Rate }=0.15\end{array}$ \\
\hline 8 & $\mathrm{MLP}_{8}$ & 2 & 13,13 & $\begin{array}{c}\text { Training_function }=\text { Traincgf } \\
\text { Learning_Rate }=0.18\end{array}$ \\
\hline 9 & $\mathrm{MLP}_{9}$ & 3 & $10,15,10$ & $\begin{array}{c}\text { Training_function }=\text { Trainlm } \\
\text { Learning_Rate }=0.15\end{array}$ \\
\hline 10 & $\mathrm{MLP}_{10}$ & 1 & 20 & $\begin{array}{c}\text { Training_function }=\text { Trainlm } \\
\text { Learning_Rate }=0.2\end{array}$ \\
\hline
\end{tabular}

\section{RESUlTS AND DISCUSSION}

The capability of the trained ANN ensemble network has been proved employing data set kept for testing i.e. test set and it has been observed that the ANN ensemble correctly classified all the 20 values of test data set into normal and abnormal classes.
The same test data set has been applied to the constituent members individually and none of the constituent members produced correct output for all the 20 inputs. This validates the proposed ANN ensemble approach for ECG classification as an accurate and effective approach. 


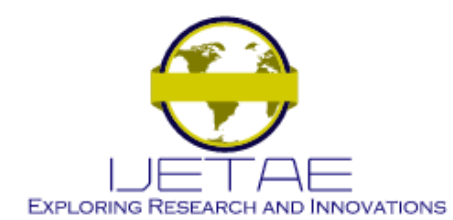

\section{International Journal of Emerging Technology and Advanced Engineering Website: www.ijetae.com (ISSN 2250-2459, ISO 9001:2008 Certified Journal, Volume 10, Issue 08, August 2020)}

The designed ANN ensemble network has been then utilized to detect the arrhythmia for 50 independent samples of ECG and it has been found that 49 samples have been correctly classified by the proposed model, thus giving an efficiency of $98 \%$. The high accuracy is due to the fact that multiple ANN models are predicting the output for same input values and it is more likely that majority of them shall produce accurate results. The implementation of the proposed approach is straightforward which makes it very effortless for implementation.

\section{CONCLUSION}

In this work, an ANN ensemble based approach has been presented to detect the arrhythmia by classifying the ECG signal into normal and abnormal classes. The ANN ensemble has been developed by employing 10 MLP constituent ANN models in parallel. The Bagging approach has been used to create diverse training data subsets to train the MLP models. The diversity among constituent ANN models has been achieved by selecting different structures and varying other parameters of the models. The overall ANN ensemble output has been decided by utilizing majority voting approach. The high accuracy observed from the classified results proves this approach as an effective approach. Also the ease in implementation makes the proposed approach a potential candidate to be employed for classification of more than 2 classes of arrhythmia.

\section{REFERENCES}

[1] Wannenburg J, Malekian R, and Hancke G P, (2018), "Wireless Capacitive-Based ECG Sensing for Feature Extraction and Mobile HealthMonitoring," IEEE Sensors Journal, 18(14), pp. 6023-32

[2] Liesbeth Mesotten, Alex Maes, Anne-Sophie Hambye, Hendrik Everaert, V. Van den Maegdenbergh, Philippe Franken and Luc Mortelmans, "Nuclear Cardiology, Part 1: Anatomy and Function of the Normal Heart," Journal of Nuclear Medicine Technology, 1998; 26:4-8

[3] Malmivuo, Jaakko \& Plonsey, Robert. (1995). Bioelectromagnetism. The Basis of ECG Diagnosis, Chap.19, pp 437-457

[4] G. Bortolan, R. Degani and J. L. Willems, "Neural networks for ECG classification," [1990] Proceedings Computers in Cardiology, Chicago, IL, USA, 1990, pp. 269-272, doi: 10.1109/CIC.1990.144212.

[5] G. E. Oien, N. A. Bertelsen, T. Eftestol and J. H. Husoy, "ECG rhythm classification using artificial neural networks," 1996 IEEE Digital Signal Processing Workshop Proceedings, Loen, Norway, 1996, pp. 514-517, doi: 10.1109/DSPWS.1996.555575.
[6] Weems A., Harding M., Choi A. (2016) Classification of the ECG Signal Using Artificial Neural Network. In: Juang J. (eds) Proceedings of the 3rd International Conference on Intelligent Technologies and Engineering Systems (ICITES2014). Lecture Notes in Electrical Engineering, vol 345. Springer, Cham

[7] T. Debnath, M. M. Hasan and T. Biswas, "Analysis of ECG signal and classification of heart abnormalities using Artificial Neural Network," 2016 9th International Conference on Electrical and Computer Engineering (ICECE), Dhaka, 2016, pp. 353-356, doi: 10.1109/ICECE.2016.7853929.

[8] Syed Muhammad Anwar, Maheen Gul, Muhammad Majid and Majdi Alnowami, "Arrhythmia Classification of ECG Signals Using Hybrid Features," Computational and Mathematical Methods in Medicine, Volume 2018, Article ID 1380348, 8 pages.

[9] T. Debnath, T. Biswas, M. H. Ashik and S. Dash, "Auto-Encoder Based Nonlinear Dimensionality Reduction of ECG data and Classification of Cardiac Arrhythmia Groups Using Deep Neural Network," 2018 4th International Conference on Electrical Engineering and Information \& Communication Technology (iCEEiCT), Dhaka, Bangladesh, 2018, pp. 27-31, doi: 10.1109/CEEICT.2018.8628044.

[10] Nurmaini, S.; Umi Partan, R.; Caesarendra, W.; Dewi, T.; Naufal Rahmatullah, M.; Darmawahyuni, A.; Bhayyu, V.; Firdaus, F. An Automated ECG Beat Classification System Using Deep Neural Networks with an Unsupervised Feature Extraction Technique. Appl. Sci. 2019, 9, 2921.

[11] Jia Li, Yujuan Si, Tao Xu, and Saibiao Jiang, "Deep Convolutional Neural Network Based ECG Classification System Using Information Fusion and One-Hot Encoding Techniques," Mathematical Problems in Engineering, Volume 2018, Article ID 7354081, 10 pages.

[12] Xiaoling Wei, Jimin Li, Chenghao Zhang, Ming Liu, Peng Xiong, Xin Yuan, Yifei Li, Feng Lin, and Xiuling Liu, "Atrial Fibrillation Detection by the Combination of Recurrence Complex Network and Convolution Neural Network," Journal of Probability and Statistics , Volume 2019, Article ID 8057820, 9 pages.

[13] Pławiak, P., Acharya, U.R. Novel deep genetic ensemble of classifiers for arrhythmia detection using ECG signals. Neural Comput \& Applic (2019). https://doi.org/10.1007/s00521-01803980-2

[14] Yang S, Browne A (2004) Neural network ensembles: combining multiple models for enhanced performance using a multistage approach. Expert Syst 21(5):279-288

[15] Yao X, Islam MM (2008) Evolving artificial neural network ensembles. IEEE Comput Intell Mag 3(1):31-42.

[16] Balwinder S. Dhaliwal and Shyam S. Pattnaik, "BFO-ANN Ensemble Hybrid Algorithm to Design Compact Fractal Antenna for Rectenna System," Neural Computing and Applications (Springer). vol. 28, no. Suppl 1, pp. S917-S928, 2017. [DOI 10.1007/s00521016-2402-9]

[17] Granitto PM, Verdes PF, Navone HD, Ceccatto HA (2002) Aggregation algorithms for neural network ensemble construction. In: Proceedings of the Brazilian symposium neural networks, pp 178-183

[18] Islam MM, Yao X, Murase K (2003) A constructive algorithm for training cooperative neural network ensembles. IEEE Trans Neural Netw 14(4):820-834 